\title{
Elastic-Arm: Human-Scale Passive Haptic Feedback for Augmenting Interaction and Perception in Virtual Environments
}

\author{
Merwan Achibet * \\ Inria Rennes, France \\ Adrien Girard * \\ Inria Rennes, France
}

\author{
Anthony Talvas * \\ INSA/Inria Rennes, France
}

\author{
Maud Marchal * \\ INSA/Inria Rennes, France
}

Anatole Lécuyer *

Inria Rennes, France

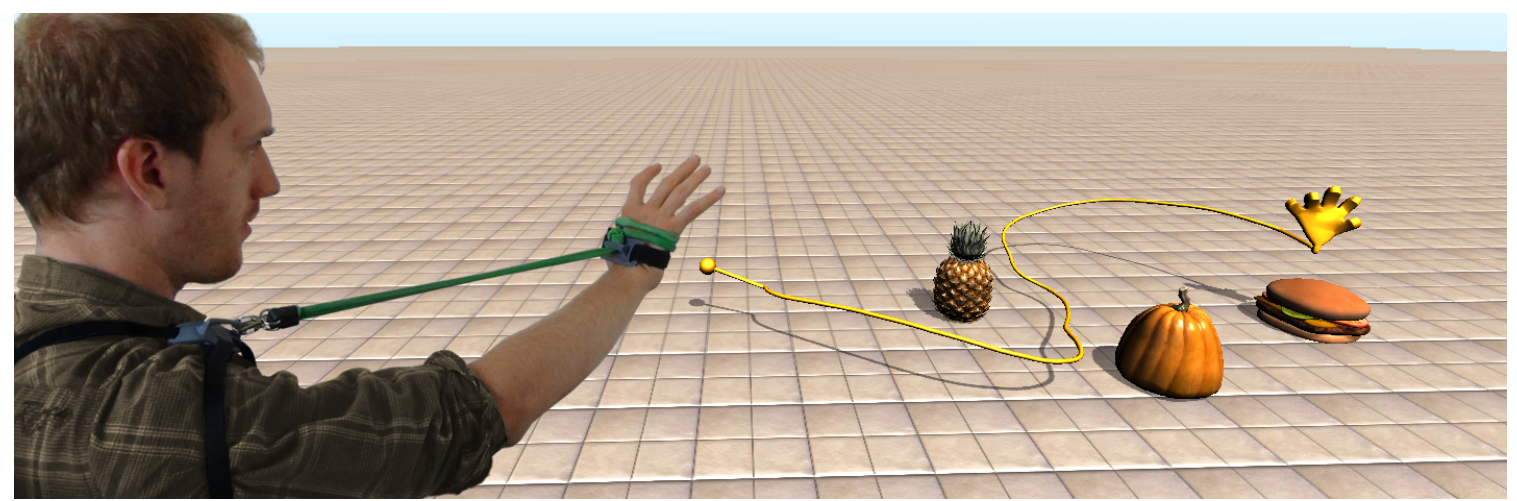

Figure 1: The Elastic-Arm is a body-mounted armature that provides egocentric passive haptic feedback. It presents an alternative to more complex active haptic devices that are generally less adapted to large immersive environments. In this example, a user performs a selection task by stretching his virtual arm using a combination of the Bubble and Go-Go techniques reimplemented with our system.

\begin{abstract}
Haptic feedback is known to improve 3D interaction in virtual environments but current haptic interfaces remain complex and tailored to desktop interaction. In this paper, we introduce the "ElasticArm", a novel approach for incorporating haptic feedback in immersive virtual environments in a simple and cost-effective way. The Elastic-Arm is based on a body-mounted elastic armature that links the user's hand to her shoulder. As a result, a progressive resistance force is perceived when extending the arm. This haptic feedback can be incorporated with various 3D interaction techniques and we illustrate the possibilities offered by our system through several use cases based on well-known examples such as the Bubble technique, Redirected Touching and pseudo-haptics. These illustrative use cases provide users with haptic feedback during selection and navigation tasks but they also enhance their perception of the virtual environment. Taken together, these examples suggest that the Elastic-Arm can be transposed in numerous applications and with various $3 \mathrm{D}$ interaction metaphors in which a mobile haptic feedback can be beneficial. It could also pave the way for the design of new interaction techniques based on "human-scale" egocentric haptic feedback.
\end{abstract}

Index Terms: H.5.2 [Information Interfaces and Presentation]: User Interfaces-Interaction styles, Haptic I/O

\section{INTRODUCTION}

Haptic feedback has been shown to improve 3D interaction in virtual environments. Even simple haptic cues, such as notifying about collisions with virtual objects, can significantly improve selection accuracy and task completion time [23, 15]. Haptic effects are also

\footnotetext{
* \{merwan.achibet, adrien.girard, anthony.talvas, maud.marchal, anatole.lecuyer\}@inria.fr
}

appreciated by users [21] and can increase the "feeling of presence" [9].

However, most existing haptic devices have been designed for desktop setups and their workspace is often limited to wrist movements [10]. Several "human-scale" haptic interfaces have been designed for larger workspaces $[5,6]$ but the resulting hardware is often expensive and cumbersome. Thus, in most cases, haptic devices limit the mobility of the users, which prevents their use in a CAVE or with a Head-Mounted Display (HMD).

Therefore, in this paper, we propose a simple and cost-effective approach for incorporating haptic feedback in immersive virtual environments. This novel approach, called "Elastic-Arm", is based on an elastic armature that is mounted on the user's shoulder and linked to her hand. In our implementation, we used simple and inexpensive components: consumer-ready arm exercisers, 3D-printed parts and a tracking system to measure the 3D positions of the two extremities of the elastic link. This setup notably provides a progressive passive haptic feedback when the user extends her arm with the goal of performing 3D interaction tasks. Such passive forcefeedback can be exploited in virtual environments for improving either 3D interaction or the perception of haptic properties of virtual objects. For instance, the Elastic-Arm can be combined with a variety of well-known interaction techniques such as the Bubble technique [4], Redirected Touching [12] or pseudo-haptics [13] in order to expand the workspace of their initial implementations, as described in this paper. Our general approach, and our novel passive haptic interface, can thus be used in a wide range of use cases in virtual reality.

In the remainder of this paper, we will first present related work on haptic interfaces and alternative means of delivering haptic feedback. In Section 3, we will describe the Elastic-Arm's concept and its current implementation. Then, in Section 4, we will present several use cases illustrating how our approach can be combined with existing and proven interactive techniques. 


\section{Related WORK}

In this section, we describe existing approaches for haptic rendering based either on active or passive interfaces and alternative haptic solutions leveraging visual feedback. We also present the results of studies that investigate the beneficial influence of haptic feedback in the context of $3 \mathrm{D}$ interaction.

\subsection{Active haptic devices}

Haptic devices vary by the degree of mobility they provide. Desktop devices are the most accessible but they suffer from small workspaces, typically limited to wrist movements [10]. They are also grounded, which limits mobility furthermore. "Human-scale" haptic interfaces have been designed to accompany users within a larger physical space [5] but they are more bulky and costly. Another option to increase user's mobility is to directly mount the interface on her body, as it is the case for haptic exo-skeletons. Such systems are however cumbersome because of their mechanical complexity and can still require to be grounded [6].

Simpler string-based interfaces have been proposed to alleviate the issues related to mechanical complexity. For instance, HapticGEAR is a backpack-like device capable of providing forcefeedback by pulling on the user's hand with strings [7]. Tsetserukou et al. [20] proposed a more minimalist haptic interface taking the form of an actuated cable that links the wrist to the arm to constrain arm extension, mimicking a muscle. While the degrees of freedom of this system are limited, it can simulate various haptic effects such as weight and collisions. These devices are more accessible thanks to simpler designs but their active nature still induces mechanical overhead and no similar wearable passive haptic devices have been proposed so far.

\subsection{Passive haptic devices}

Insko [9] defined passive haptics as : "a technique that incorporates passive physical objects into virtual environments to physically simulate the virtual objects". Therefore, passive interfaces are replicas of virtual objects that provide feedback through their shape. Examples of such passive feedback include using a physical ledge on the floor to recreate the haptic sensation of standing on the edge of a hole [9] and using a panel made of wood to simulate the tactile feedback of a control panel [2]. Similarly, Poupyrev et al. [18] used a tangible tablet to reproduce the feedback and support of a notepad in a virtual handwriting task.

A second definition is proposed by Swanson [19]: passive haptic devices are "interfaces [that] use energetically passive actuators which may in general only remove, store, or redirect kinetic energy within the system". Based on this definition, users are the source of energy and the system can only dissipate, restore or steer that energy. Several haptic devices that fit Swanson's definition have been proposed. For instance, Pihuit et al. [16] and Achibet et al. [1] respectively used foam balls and hand exercisers as proxies to simulate the grasping of objects. Hoang et al. [8] proposed to use a passive deformable glove to perform 3D modeling tasks. Paljic et al. [14] also developed a passive stringed system with user-actuated brakes to display a stickiness sensation. Through various form factors, these passive devices demonstrated that, even with simple designs, they were able to simulate advanced haptic effects.

\subsection{Vision-based alternatives to haptic feedback}

A main issue of passive haptic devices is their low flexibility to support different environments and scenarios. Alternative techniques relying on the dominance of vision over proprioception have been designed to address this issue.

Kohli et al. [12] presented Redirected Touching; a spatial warping that leverages discrepancies between the haptic feedback of the real world and the visual feedback of the virtual world in order to distort shape perception. They compared passive haptic feedback with and without Redirected Touching and few participants realized the mismatch between visual and haptic feedback.

Another solution to extend the possibilities of passive devices is pseudo-haptic feedback, as introduced by Lécuyer et al. [13]. They initially introduced a dynamic mismatch between the force applied on an isometric device and the corresponding movement of the virtual object being manipulated (the Control/Display ratio). This effect was illustrated with a task involving the insertion of an object into a duct: when the manipulated object reaches the duct, the movement is slowed down and users increase the force applied on the passive device which makes them perceive a friction force. Pseudo-haptic feedback can also simulate other haptic properties such as mass [3] and grasping forces [1].

These haptic effects expand the capabilities of passive devices without relying on complex mechanical systems. The approach that we propose in this paper, the Elastic-Arm, is motivated by such effects and aims at providing a similarly convincing feedback with simple and accessible hardware.

\subsection{Benefits of haptic feedback}

Several user studies investigated the beneficial effects of active force-feedback and showed that it can indeed improve accuracy in selection tasks [23] as well as reduce task completion time [15].

The effects of passive haptic feedback have also been specifically investigated. Insko [9] investigated the influence of passive haptics in a pit hole scene where feedback was supported by a physical ledge on the floor. Their experiment shows through physiological measures that passive haptics enhances the sense of presence. Viciana-Abad et al. [22] investigated a task consisting in playing with a virtual Simon game and confirmed the increase of sense of presence. They also showed that passive haptics improves task performance by reducing both execution time and number of errors. Hoang et al. [8] showed that their passive glove enhanced user's performance and reduced fatigue in a modeling task. Unlike the previous studies, Borst and Volz [2] compared active and passive feedback in a task consisting of interacting with a virtual control panel. This experiment showed that there were no significant differences in performance between passive and active feedback for their task.

\section{The Elastic-Arm}

Concept - The system that we propose is a novel bodymounted elastic armature that enhances interaction in virtual environments by providing passive haptic feedback to the user's arm. It relies on an elastic cable that links the user's hand to her shoulder (Figure 2a). When she stretches out her arm in order to perform interaction tasks, she can thus perceive an effort proportional to the elastic's stiffness. This egocentric resistance force can then be leveraged in order to incorporate haptic feedback either into interaction techniques without any haptic components or into interaction techniques originally relying on active haptic devices or passive props.

Implementation - For providing the essential haptic feedback of the Elastic-Arm, elastic cables sold as arm exercisers were used. Each end of the elastic cable is attached through a hook to 3Dprinted straps; one on the shoulder and the other on the back of the hand. Velcro bands wrapped around the arm and around the torso ensure that the shoulder strap remains securely fixed when the user stretches her arm and pulls the elastic cable.

As illustrated in Figure 2b, this setup inherently creates a dichotomy between two states: when the elastic is relaxed and when it is taut. The transition between these states can be controlled by the user by extending her arm as well as gauged thanks to the haptic feedback provided. Designing interaction techniques around this feature requires both the position of the hand $\boldsymbol{h}$ and the position of the shoulder $\boldsymbol{s}$ in order to obtain the reach vector $\boldsymbol{r}=\boldsymbol{h}-\boldsymbol{s}$. In our 


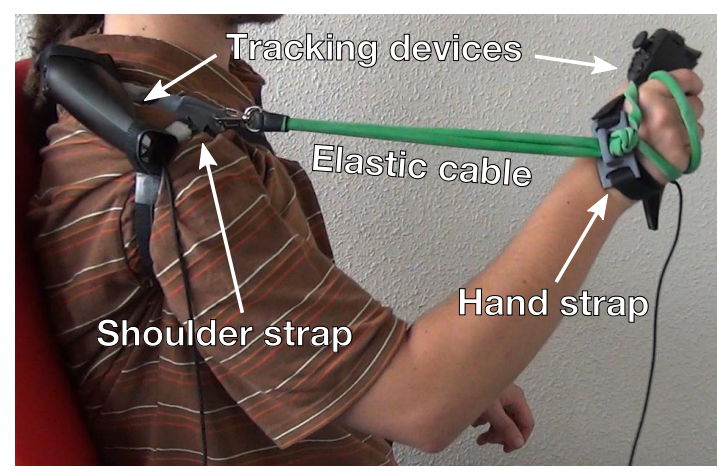

(a)

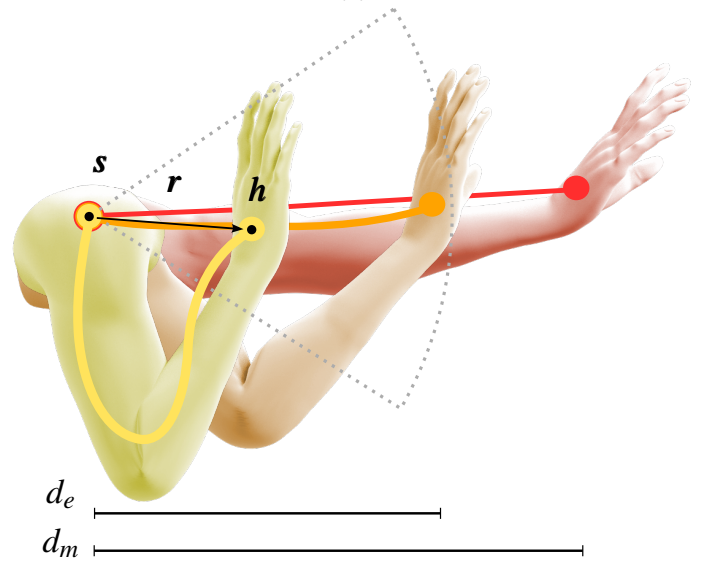

(b)

Figure 2: The Elastic-Arm. (a) The armature is composed of an elastic cable (an arm exerciser in our prototype), 3D-printed straps and tracking hardware (a Razer Hydra in our prototype). (b) Different states: relaxed cable (yellow), nearly taut (orange) and extended arm (red). The dotted radius corresponds to the spherical boundary of the elastic cable beyond which haptic feedback is felt $\left(\|\boldsymbol{r}\|>d_{e}\right)$.

prototype, a Razer Hydra ${ }^{1}$ was used for tracking these positions. However, any other tracking system for desktop interaction or for large physical spaces can also be used; camera-based setups for instance.

The rest length of the elastic cable is defined as $d_{e}$. Thus, when the hand is closer to the body $\left(\|\boldsymbol{r}\|<d_{e}\right)$, the elastic cable is not taut and the user's arm moves in an unconstrained manner. However, when $\|\boldsymbol{r}\|>d_{e}$, an effort proportionate to the cable's stiffness pulls the arm back. Another potentially useful value is the maximum reach of the user's arm $d_{m}$. These thresholds can be obtained through a short calibration step by, first, asking the user to stretch her arm until the elastic is taut $\left(d_{e}\right)$ and then asking her to stretch her arm as much as possible $\left(d_{m}\right)$. The thresholds can then be exploited for the design of relevant control schemes.

Discussion - The design of the Elastic-Arm is motivated by established literature showing that passive haptic feedback is involved in enhancing both performance $[2,22]$ and perception $[13$, $14,11]$ and provides a stronger sense of presence $[9,22]$. Through this system, our aim is to provide a mobile, low-cost and easily reproducible mean to leverage such an essential feedback. Thus, existing interaction techniques could be augmented with the use of our system; which additionally opens the gate for all-new interaction methods specifically designed around its egocentric haptic capabilities.

\footnotetext{
${ }^{1}$ http://sixense.com/razerhydra
}

In order to illustrate the possibilities offered by the Elastic-Arm, Section 4 presents several application examples, focused on control and perception. For these illustrative use cases, we chose to leverage the virtual hand metaphor since it provides an engaging and meaningful representation for descriptive purposes. This metaphor also fits well with the egocentric nature of the Elastic-Arm. Therefore, the associated demo applications display a bright orange hand controlled by the user (inspired by Mr. Tickle, a cartoon character capable of stretching his arms to perform various feats). In a more general perspective however, the Elastic-Arm could be used in conjunction with a variety of other interaction modalities.

\section{Illustrative use cases}

In this section, we present several illustrative use cases based on existing interaction techniques. These techniques were originally designed and evaluated with haptic feedback in mind and rely on grounded haptic devices, either active or passive. Here, we adapt them to the Elastic-Arm in order to demonstrate how our system can make such methods egocentric and more mobile whilst providing a similar haptic feedback.

These use cases are divided into two categories. Firstly, we explore how interaction tasks such as object selection and navigation can benefit from the Elastic-Arm. Then, we explore perceptual effects made possible by our system and propose examples of approaches for enhancing the users' perception of a virtual environment.

\subsection{Using the Elastic-Arm to improve 3D interaction}

Here, we propose two illustrative use cases that enable (1) selection of distant objects by stretching a virtual arm and (2) navigation towards out-of-view areas in order to reach occluded objects.

\subsubsection{Selection of distant objects with the Bubble technique}

The first example that we propose enables users to select virtual objects that are out of reach by stretching their virtual arm. This example is based on the hybrid position/rate control of the Bubble technique [4]. It is also reminiscent of the Go-Go technique [17] with regards to its arm-extension mechanics.

In this scenario, users control a virtual hand and select objects by touching them. Similarly to the Bubble technique, two control modes can be differentiated. To begin with, the virtual hand is position-controlled with a 1:1 mapping if $\|\boldsymbol{r}\|<d_{e}$, which is relevant when the user performs interaction tasks in her close vicinity. However, when the elastic cable is taut $\left(\|\boldsymbol{r}\| \geq d_{e}\right)$, the virtual hand switches to rate-control and stretches to reach faraway targets (Figure 3a). Flexing the arm below the elastic threshold at any moment triggers a rewinding animation that quickly rolls the arm backward to its initial position.

In rate-control mode, when the virtual arm stretches, users have control over the speed of the virtual hand by extending their arm farther away from their shoulder. They also steer the hand by pointing their arm in the direction that they wish to go. These relations are described in the following equation and Figure $3 \mathrm{~b}$, with $\boldsymbol{h}_{\boldsymbol{v}}$ the position of the virtual hand and $k_{p}$ a scaling coefficient:

$$
\boldsymbol{h}_{v}(t+1)=\boldsymbol{h}_{v}(t)+\hat{\boldsymbol{r}}\left(\|\boldsymbol{r}\|-d_{e}\right) k_{p}
$$

This implementation of the Bubble technique with the ElasticArm shares similar principles with its original version that made use of active haptic feedback. Distant targets can also be reached by leveraging an hybrid control scheme. However, even without an active device, the interaction is still assisted by the same feedback. Notably, the transition between position and rate control (the bubble's boundaries) can be perceived by users. Similarly, the haptic feedback perceived when stretching the virtual hand is proportional to its speed. As shown by Zhai [24], rate-control provides better performance with self-centering elastic devices such as ours. Thus, 
the Elastic-Arm seems well adapted to control schemes like that of the Bubble.

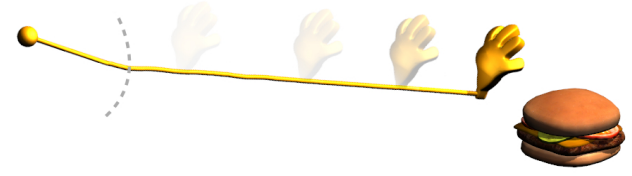

(a)

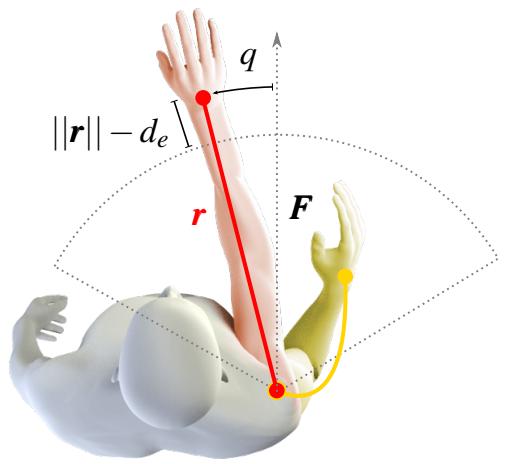

(b)

Figure 3: Selecting out-of-reach objects. (a) In rate-control mode, the virtual arm stretches to reach faraway targets beyond the limit of the user's real reach (gray dotted line). (b) The control mode depends on the extension of the arm: the virtual hand is position-controlled below the elastic threshold (yellow) and the hand is rate-controlled beyond the elastic threshold (red).

\subsubsection{Navigation and selection of occluded objects with the BubbleCam technique}

We propose a variation of the previous method that additionally enables to select occluded objects by navigating across the virtual environment and around obstacles. The associated controls are similar to the previous method but the displayed view is similar to the Bubble Cam technique [4].

Here, the virtual camera displaying the scene is fixed to the virtual hand. In this way, the precision of the control does not decrease as the hand moves away since the user keeps the same relative viewpoint. In order to accommodate the egocentric nature of the ElasticArm, we also complemented the Bubble's original control scheme with rotations: users can rotate their virtual hand by pointing away from the forward direction $\boldsymbol{F}$. These relations are summed up by the following equations and Figure $3 \mathrm{~b}$, with $\boldsymbol{h}_{\boldsymbol{v}}$ and $o_{v}$ the position and orientation of the virtual hand, $k_{p}$ and $k_{o}$ scaling coefficients and $q$ the quaternion representing the rotation from $\boldsymbol{F}$ to $\boldsymbol{r}$ :

$$
\boldsymbol{h}_{v}(t+1)=\boldsymbol{h}_{v}(t)+\hat{\boldsymbol{F}}\left(\|\boldsymbol{r}\|-d_{e}\right) k_{p} \quad \boldsymbol{o}_{v}(t+1)=\boldsymbol{o}_{v}(t) q^{k_{o}}
$$

The combination of a fixed camera and rotation controls enables to navigate along more intricate paths in order to reach occluded objects. For instance, Figure 4 illustrates how an object hidden behind a wall can be selected. For this illustrative method to provide true navigation capabilities, users are also able to set the current position of the hand as a new starting position by pressing a button (the trigger of the Razer Hydra in our prototype). In this way, they can navigate to different points of interest and then stretch their arm to grab objects before rolling back to this chosen position.

\subsection{Using the Elastic-Arm to improve perception}

The second set of illustrative use cases that we propose explores how the Elastic-Arm can improve user's perception of the virtual

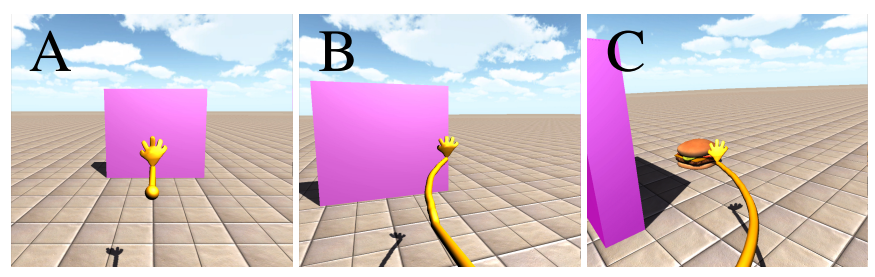

(a)

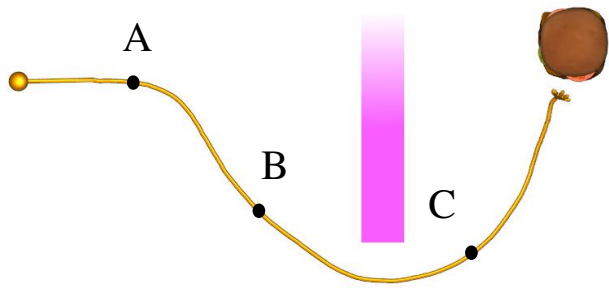

(b)

Figure 4: Navigating to out-of-view areas. (a) Successive frames of the user's view when reaching for an occluded object. The camera follows the virtual hand to maintain a constant level of precision. (b) Top view of the path traveled by the virtual hand.

environment. Two different examples are proposed: (1) a redirection effect to touch the virtual environment through our elastic armature and (2) a pseudo-haptic effect allowing users to perceive varying levels of effort when interacting with virtual objects.

\subsubsection{Perception of the virtual environment boundaries with Redirected Touching}

For this illustrative use case, our aim is to provide users with haptic cues related to the physical bounds of the virtual environment so that they can perceive its limits by probing their vicinity.

This example is inspired by Redirected Touching [12], an haptic effect that leverages a passive prop and alters its virtual appearance as well as the position of the users' hand on its surface to make them perceive a different shape when they touch it. Similarly, the method proposed here relies on a discrepancy between visual and real positions: we alter the position of the user's virtual hand so that its encounter with an obstacle is correlated with the elastic cable being taut. In this way, users are able to perceive a clear resistance when "touching" virtual objects. Since this effect relies on a visual discrepancy, users wear a HMD so that only their virtual hand is visible.

The alteration of the virtual hand's position consists in varying its Control/Display ratio with respect to the distance to facing obstables. In order to obtain this distance $d_{o}$, rays are continuously cast in the $\boldsymbol{r}$ direction. The Control/Display ratio between real and virtual hands is then adjusted so that the cable tension matches the potential collision with the obstacle, as illustrated in Figure 5a. In other words, the distance to the obstacle is mapped on the rest length of the elastic cable. The following equation sums up this principle ( $\boldsymbol{s}$ is the shoulder position):

$$
\boldsymbol{h}_{v}(t)=\boldsymbol{s}+\hat{\boldsymbol{r}} d_{o} \min \left(1, \frac{\|\boldsymbol{r}\|}{d_{e}}\right)
$$

Figure $5 b$ presents a scene featuring this redirection effect. Users are presented with a virtual cockpit and they can touch its different parts. The Elastic-Arm, associated with the technique presented in this section, then lets them perceive the collisions of the virtual hand with the cockpit elements. This application of the idea of Redirected Touching with the Elastic-Arm enables to feel virtual obstacles that are within the user's reach. However, contrary to the original implementation, users do not have to stay in front of a 

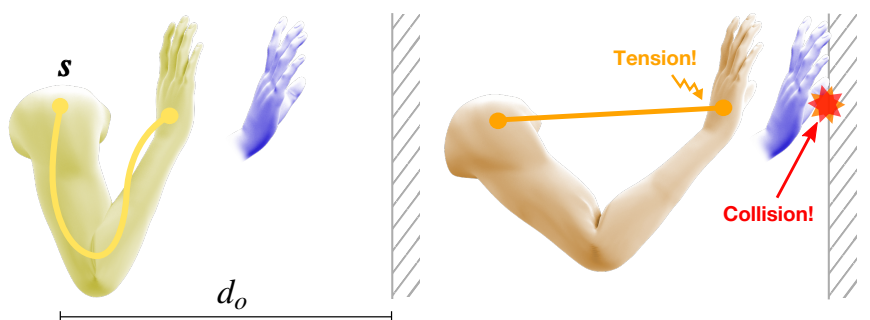

(a)
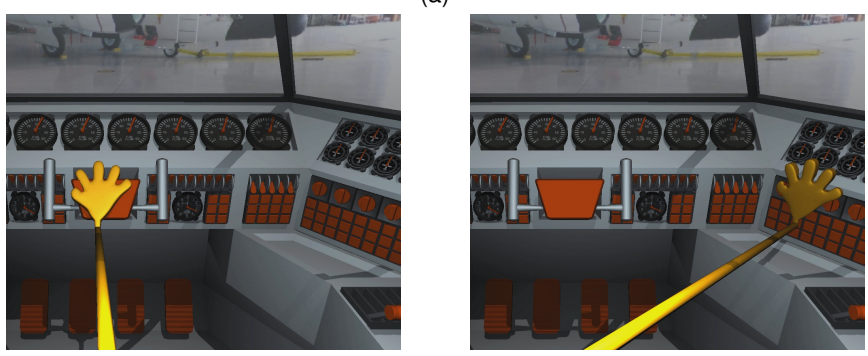

(b)

Figure 5: Probing the boundaries of the virtual environment. (a) Left: the virtual hand (blue) does not collide with any obstacle yet and the user's arm is below the elastic threshold; there is no feedback. Right: the virtual hand now collides with an obstacle. The Control/Display ratio was adjusted to match this event with the tension of the elastic cable and a resistance is felt. (b) Users can probe the boundaries of a virtual cockpit and "touch" objects at various distances like the rudder or the control panels.

grounded passive prop since the haptic feedback is here provided by the body-mounted armature. In principle, this technique could thus make a large virtual environment tangible.

\subsubsection{Perception of variable levels of effort with pseudo- haptics}

The second perception-oriented use case that we present is based on pseudo-haptics [13] and leverages the Elastic-Arm to simulate different levels of effort when interacting with virtual objects.

Pseudo-haptics is an alternative means of delivering haptic sensations that simulates haptic properties by relying on visual feedback coupled with the motion and actions of the user. Here, we apply this principle and alter the speed of the user's virtual hand depending on the haptic properties of the object it is interacting with. In this way, users have to stretch their arm to different degrees depending on the object. In consequence, users perceive different levels of effort thanks to the elastic nature of our armature.

This effect builds on the previous technique based on Redirected Touching, since the virtual hand must first collide with an object and the elastic cable must be just taut in order to start the effect. Then, once the interaction begins, the motion of the virtual hand is altered depending of the object's properties. In practice, each object is associated with a different interaction coefficient $k_{i}$ that governs how the motion of the virtual hand is scaled during the interaction ( $k_{i} \in\left[0,1\left[\right.\right.$ slows down the motion, $\left.k_{i} \in\right] 1, \infty[$ amplifies it). The following equation describes this principle:

$$
\boldsymbol{h}_{\boldsymbol{v}}(\boldsymbol{t})=\boldsymbol{s}+\hat{\boldsymbol{r}} d_{o} \min \left(1, \frac{\|\boldsymbol{r}\|}{d_{e}}\right)+\hat{\boldsymbol{r}} k_{i} \frac{\|\boldsymbol{r}\|-d_{e}}{d_{m}-d_{e}}
$$

Through this effect, users can interact with different objects and feel different levels of effort. In Figure 6a, two deformable objects are pushed on and the one with the smaller $k_{i}$ bends to a lesser degree, even whilst the arm is stretched more, and the resulting haptic feedback is greater. Figure $6 \mathrm{~b}$ shows a demonstration application
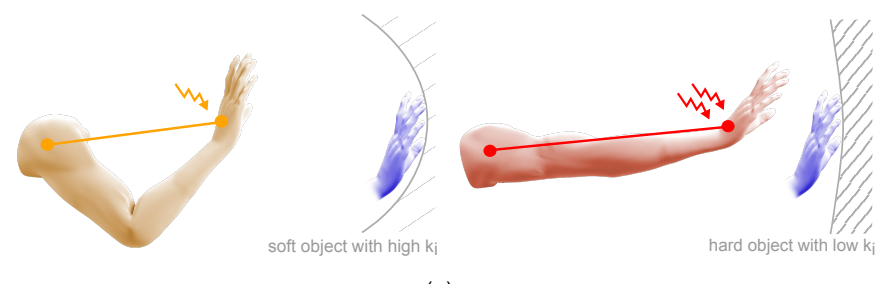

(a)
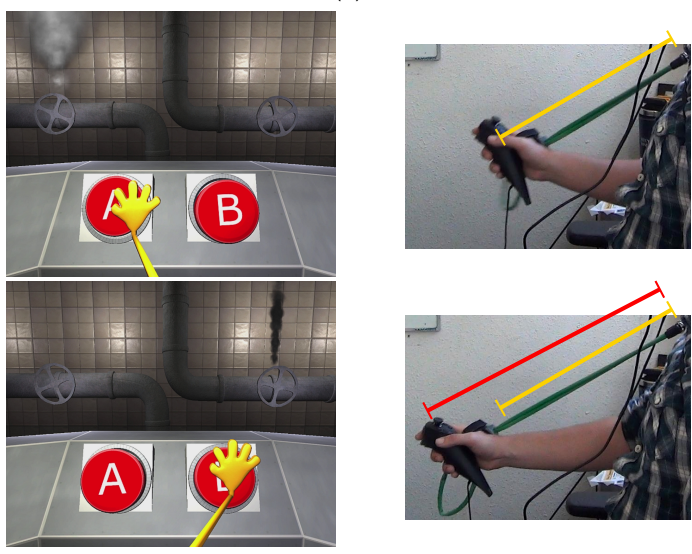

(b)

Figure 6: Simulating different levels of effort. (a) Two different deformable objects are interacted with. Left: the object is soft so the hand motion is amplified and the user moderately stretches her arm to bend the surface; the haptic feedback is moderate. Right: the object is stiff so the hand motion is slowed down and the user must stretch her arm to a greater degree; the haptic feedback is stronger. (b) Users have to stretch their arm to a greater degree to interact with the rightmost button and the haptic feedback is greater.

in which users can push two different buttons and perceive different effort requirements to activate them. More generally, this effect could simulate various haptic properties related to pushing actions; closing a drawer or pushing on a wheeled cart, for instance.

\section{Discussion}

The illustrative use cases that we presented explored how the Elastic-Arm could apply to proven interaction techniques, both in terms of control and in terms of perception. Future work could consist in designing full-fledged interaction techniques from the ideas explored in this paper and then assess their effect on performance and perception in specific application contexts. We could especially investigate the possibilities offered by the Elastic-Arm when used in large physical spaces.

Indeed, compared to desktop haptic devices with limited clearance, the Elastic-Arm provides an extended workspace. Since, it is always centered on the user's shoulder, the workspace is by design as large as the user's reach. Moreover, unlike with desktop devices or static passive props, users do not have to keep the same fixed position and they are free to move around a larger physical space such as an immersive CAVE.

Another convenience of the Elastic-Arm is its simple and reproducible design. Its components (elastic cable, 3D-printed straps) make it easy to build. The elastic cable can be found in a variety of lengths to accommodate different user morphologies. Similarly, it can be found with different levels of stiffness depending on the targeted application context. This makes the Elastic-Arm an affordable and versatile system that could find uses in many domains.

Alternative designs of the Elastic-Arm could be considered. With the current prototype, the magnitude of the haptic feedback 
is limited to the stiffness of the elastic cable used. However, more elaborate rigs with several elastic cables of different lengths could be attached on the same arm. In this way, more "layers" of stiffness could divide the user's reach.

Currently, the haptic feedback is unidirectional, in the sense that it is always directed towards the user's shoulder. Another way to provide a richer haptic feedback could be to consider other anchor points for the elastic cables. For example, another cable could link the waist to the hand. With such setups, the development of other haptic effects, like gravity, could be explored. Then, the coexistence of several elastics and the potential interferences that could occur between them should be investigated.

We could also consider equipping more body parts with similar elastic rigs. For instance, fingers could be connected by short elastic cables in order to design new manipulation techniques enhanced by a portable passive feedback. While the hand is a major tool for 3D interaction, less exploited body parts could be considered too and another possibility would be to equip the legs with similar elastic armatures. In this specific instance, novel navigation techniques based on the provided haptic feedback could be designed. From a more general perspective, designing such localized elastic armatures could lead to the development of an "elastic exoskeleton" and make general-purpose haptic feedback more accessible for virtual reality applications.

\section{Conclusion}

In this paper, we proposed the Elastic-Arm, a body-mounted armature providing passive haptic feedback to the user's arm. The simple design of our system enables haptic feedback within a large workspace compared to more complex and more costly active haptic devices and compared to static passive props. Several illustrative use cases were also presented to illustrate the capabilities of our system. First, two examples focused on interaction allowed respectively to select distant virtual objects and to navigate in a virtual environment to grab occluded objects. Here, the Elastic-Arm provided a cost-effective alternative to active haptic devices and extended the physical workspace. Then, two use cases focused of user's perception and delivered haptic cues respectively about the boundaries of the virtual environment and about the haptic properties of manipulated objects. Here, the Elastic-Arm provided passive feedback without relying on static props, thereby extending the user's mobility. These illustrative use cases were chosen examples but many other interaction techniques for performing a wide variety of tasks could be adapted too. For instance, the use of the ElasticArm for object manipulation or system control could be explored.

This work leads us to believe that the Elastic-Arm could be incorporated in a wide range of contexts. For instance, perceptionoriented methods such as those presented in this paper could be used for ergonomics studies. Another field of application that would fit the Elastic-Arm is medical rehabilitation. Tasks of increasing difficulty performed with armature of different stiffnesses could for example be proposed to patients in order to progressively enhance their physical performance.

\section{ACKNOWLEDGEMENTS}

This work was supported by ANR (MANDARIN project, ANR-12CORD-0011).

\section{REFERENCES}

[1] M. Achibet, M. Marchal, F. Argelaguet, and A. Lécuyer. The virtual mitten: A novel interaction paradigm for visuo-haptic manipulation of objects using grip force. In Proc. of 3DUI, pages 59-66, 2014.

[2] C. Borst and R. Volz. Evaluation of a haptic mixed reality system for interactions with a virtual control panel. Presence, 14(6):677-696, 2005.
[3] L. Dominjon, A. Lécuyer, J. Burkhardt, P. Richard, and S. Richir. Influence of control/display ratio on the perception of mass of manipulated objects in virtual environments. In Proc. of $V R$, pages 19-25, 2005.

[4] L. Dominjon, A. Lécuyer, J.-M. Burkhardt, and S. Richir. A comparison of three techniques to interact in large virtual environments using haptic devices with limited workspace. In Proc. of Computer Graphics International Conference, volume 4035, pages 288-299. 2006.

[5] L. Dominjon, J. Perret, and A. Lécuyer. Novel devices and interaction techniques for human-scale haptics. The Visual Computer, 23(4):257266, 2007.

[6] A. Gupta and M. K. O'Malley. Design of a haptic arm exoskeleton for training and rehabilitation. Trans. Mechatronics, 11(3):280-289, 2006.

[7] M. Hirose, K. Hirota, T. Ogi, H. Yano, N. Kakehi, M. Saito, and M. Nakashige. HapticGEAR: the development of a wearable force display system for immersive projection displays. In Proc. of $V R$, pages 123-129, 2001.

[8] T. N. Hoang, R. T. Smith, and B. H. Thomas. 3D interactions with a passive deformable haptic glove. In Proc. of ISMAR, pages 1-6, 2013.

[9] B. E. Insko. Passive Haptics Significantly Enhances Virtual Environments. PhD thesis, The University of North Carolina at Chapel Hill, 2001.

[10] H. Iwata. Artificial reality with force-feedback: development of desktop virtual space with compact master manipulator. In Proc. of $S I G$ GRAPH, pages 165-170, 1990.

[11] L. Kohli. Redirected touching: Warping space to remap passive haptics. In Proc. of 3DUI, pages 129-130, 2010.

[12] L. Kohli, M. C. Whitton, and F. P. B. Jr. Redirected touching: The effect of warping space on task performance. In Proc. of 3DUI, pages 105-112, 2012.

[13] A. Lécuyer, S. Coquillart, A. Kheddar, P. Richard, and P. Coiffet. Pseudo-haptic feedback: Can isometric input devices simulate force feedback? In Proc. of VR, pages 83-90, 2000.

[14] A. Paljic and S. Coquillart. A passive stringed haptic system for immersive environments. In Proc. of EuroHaptics, pages 82-87, 2004.

[15] V. Pawar and A. Steed. Evaluating the influence of haptic forcefeedback on 3D selection tasks using natural egocentric gestures. In Proc. of VR, pages 11-18, 2009.

[16] A. Pihuit, P. Kry, and M.-P. Cani. Hands on virtual clay. In Proc. of Shape Modeling and Applications, pages 267-268, 2008.

[17] I. Poupyrev, M. Billinghurst, S. Weghorst, and T. Ichikawa. The go-go interaction technique: Non-linear mapping for direct manipulation in vr. In Proc. of UIST, pages 79-80, 1996.

[18] I. Poupyrev, N. Tomokazu, and S. Weghorst. Virtual notepad: handwriting in immersive VR. In Proc. of VRAIS, pages 126-132, 1998.

[19] D. K. Swanson. Implementation of Arbitrary Path Constraints using Dissipative Passive Haptic Displays. PhD thesis, School of Mechanical Engineering, Georgia Institute of Technology,, 2002.

[20] D. Tsetserukou, K. Sato, and S. Tachi. Exointerfaces: Novel exosceleton haptic interfaces for virtual reality, augmented sport and rehabilitation. In Proc. of Augmented Human, pages 1-6, 2010.

[21] L. Vanacken, C. Raymaekers, and K. Coninx. Evaluating the influence of multimodal feedback on egocentric selection metaphors in virtual environments. In Proc. of Haptic and Audio Interaction Design, pages 12-23. 2006.

[22] R. Viciana-Abad, A. R. Lecuona, and M. Poyade. The influence of passive haptic feedback and difference interaction metaphors on presence and task performance. Presence, 19(3):197-212, June 2010.

[23] S. A. Wall, K. Paynter, A. M. Shillito, M. Wright, and S. Scali. The effect of haptic feedback and stereo graphics in a 3D target acquisition task. In Proc. of Eurohaptics, 2002.

[24] S. Zhai. User performance in relation to 3D input device design. Proc. of SIGGRAPH, 32(4):50-54, 1998. 